\title{
Tracking Outfield Employees using GPS in Web Applications
}

\author{
Vinothini Kasinathan ${ }^{1, \star}$, Aida Mustapha ${ }^{2}$, and Roshein Chandran ${ }^{1}$ \\ ${ }^{1}$ Faculty of Computing, Technology and Engineering, Asia Pacific University of Innovation and Technology, Technology Park \\ Malaysia, Bukil Jalil, Kuala Lumpur 57000, Malaysia \\ ${ }^{2}$ Faculty of Computer Science and Information Technology, Universiti Tun Hussein Onn Malaysia, 86400 Parit Raja, Batu Pahat, \\ Johor, Malaysia
}

\begin{abstract}
This paper presents e-Track, a web-based tracking system for outfield employees in order to cater for various business activities as demanded by the business owners. Such demands may range from a simple task assignment, to employee location tracking and remote observation of the employees' task progress. The objective of the proposed system is two-fold. First, the employees to access the application and clocks-in work. Second, a standalone web system for the employers to determine the approximate location of the staff assigned with outfield duties. The IP address recognition will ensure no buddy punching takes place. e-Track is hoped to increase efficiency among employees by saving time travelling between branches during outfield duties. In the future, e-Track will be integrated with claim and payment modules to support arrangement for outfield duties.
\end{abstract}

\section{Introduction}

Business owners are now looking for more ways to incorporate technology to their benefit. Each business has to have its own set of employees for a company to maintain sustainability. It is worth noting that running a business is a risky venture to which a survey attests to proving $49 \%$ of businesses fail within their first five years and approximately $30 \%$ of businesses don't even make it through the first two years [1]. One of the reasons as to why businesses fail, especially small businesses are due to a lack of employee productivity [2].

There are several problems employers and employees face which reduces their productivity in a working environment. In most organizational setting, there are no standard methods to track employee with outfield works that actually meets a business's needs. The way the working environment functions currently would not be optimal to nurture an individual's full potential. Employees have to check in to work using punch cards and would have to stay on in their office cabin throughout their working hours [3]. Even if an employee has outfield duties, it is a complicated procedure for an employee to travel to a destination and return to office to sign out before office hour ends. The scenario worsens if the company has multiple offices and employees have to be travelling constantly.

Another issue is buddy punching. According to Synerion[4], buddy punching is described as when one individual checks in for another colleague of his without them being there. This is a wastage of company resources as each employee are being paid accordingly. This issue may lead to employee dissatisfaction, as some employees

\footnotetext{
^e-mail: vinothini@apu.edu.my
}

who give full effort and those who slack off get paid the same amount. In the long run, there is a high chance of employee productivity decreasing. It is clear that the traditional method used to track employee activities based on card swipes or manual entry using a book keeping method is no longer effective.

To address the issues, a Web-based Outfield Employee Tracking System named e-Track is proposed. e-Track architecture is proposed to be on a client server basis whereby there will be a mobile application for employees to access and a standalone web application for the employers to determine the approximate location of the staff assigned with outfield duties. Any organization that sends their employees for outfield duties will find this application extremely useful as they would be able to assign duties to employees based on their locations. In addition, this application also allows companies with many branches to take advantage of this system by allowing checking in to work through the mobile application. The IP address recognition will ensure no buddy punching takes place. The advantages are mutually beneficial as employees will also feel secure that their whereabouts are known by the employee in case of any mishaps taking place.

The remainder of this paper is as follows. Section 2 presents the related works, Section 3 presents the architecture and prototype of the proposed system e-Track, and finally Section 4 presents discussions and conclusions with plans for future work.

\section{Related Work}

In this modern era, tracking devices have becoming a common household gadget in human daily lives for var- 
ious reasons, ranging from tracking missing pets to missing phones. The devices are small and convenient to use. Nonetheless, tracking devices have not been completely utilized in an organizational setting. In this section, three online time tracking applications are reviewed, which are TSheets, ActivTrak, and Veriato 360.

\subsection{TSheets}

TSheets is the mobile applications name wherein it allows users to track time spent from any location, using any device in real time [5] (Refer Figure 1). This application would be useful for employees who work in remote locations or switch jobs regularly. Employees would be able to track time easily as there is clock in and clock out reminders, employee breaks and overtime alerts as well to ensure all employees are aware and up to date of the current situation ongoing in the company. The main functionality of TSheets is the use of Global Positioning System (GPS) for online time tracking. The location at which the employee has clocked-in is visible with the aerial view.

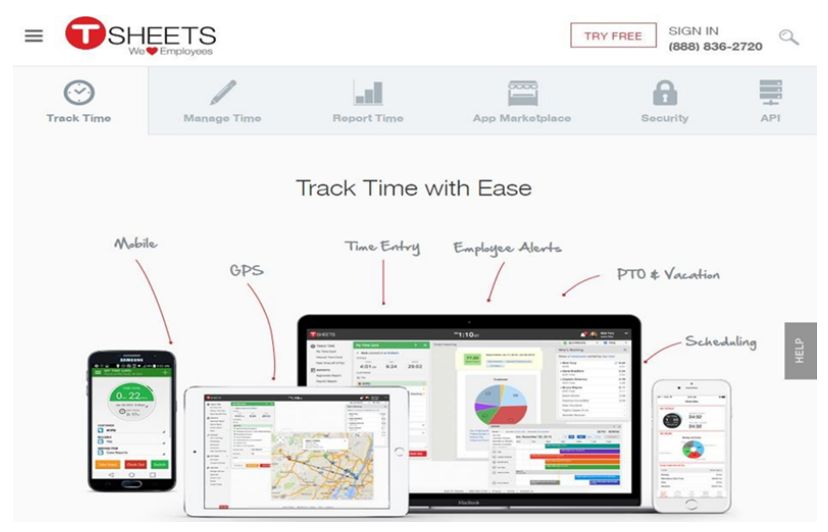

Figure 1. User interface for TSheets Time Tracking Source.

Secondly, the time entry can be manual, punch or custom. GPS location points are immediately attached to the employee's timesheet when they $\log$ in and $\log$ out or even change job modes. Employees are allowed to log in and $\log$ out at real time. On extenuating circumstances, employees can enter their time manually and allocate time to custom projects and tasks. Finally, in the case of any absenteeism such as sick days, personal days or even a family vacation, TSheets tracks employee time off to manage employees paid time off. This function enables employees to request time offs directly from the mobile application and employers would be able to approve the request in a similar manner.

\subsection{ActivTrak}

ActivTrak is a free employee monitoring software and it is developed on the basis of a free cloud-based monitoring service [6] (Refer Figure 2). It allows employers to know who is doing what and for how long by tracking applications and web usage based on each workstations. ActivTrak has three main features, which are real-time monitoring, alarm, and website blocking.

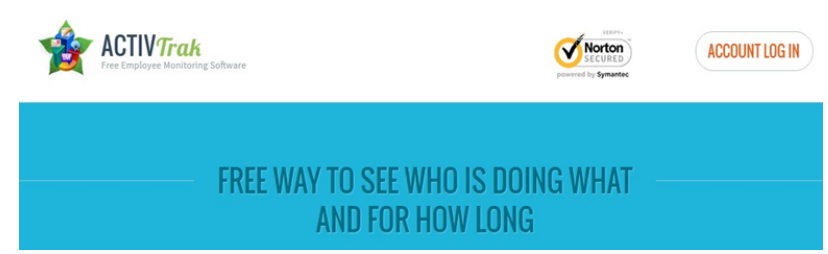

Figure 2. ActivTrak homepage.

For real-time monitoring, ActivTrak allows administrators to view a live data stream of the active window on any of the monitored devices screen. The administrators are able to see the time, device, user, title and even its public IP address. With real time monitoring, ActivTrak provide details on exactly the events happening in the monitored devices at any point from anywhere.

ActivTrack also has alarms that can be set to trigger at any actions of employees. For example, if an employee visits any restricted websites for longer than 20 seconds, an alarm will ring indicating them to close the tab. Each time an alarm is triggered, there is an option to take multiple screenshots of the complete screen to have a proof of evidence. The admin may also opt to display a popup message which would appear on the screen of the user. If all else fails and employees are still on the restricted website, there is an option to terminate the application which triggered the alarm.

Finally, websites which employers feel takes the employees productivity away can be blocked. A website can be blocked by entering the domain name and clicking the 'blocked column'. Websites can be blocked for individual users, groups or even everyone at once. Thus, there will be no complications if separate groups of people must be blocked from specific websites.

\subsection{Veriato 360}

Veriato 360 is a record-keeping system wherein it presents detailed, accurate and actionable data used in incident response together with high-risk insider monitoring and productivity reporting [7] (Refer Figure 3).

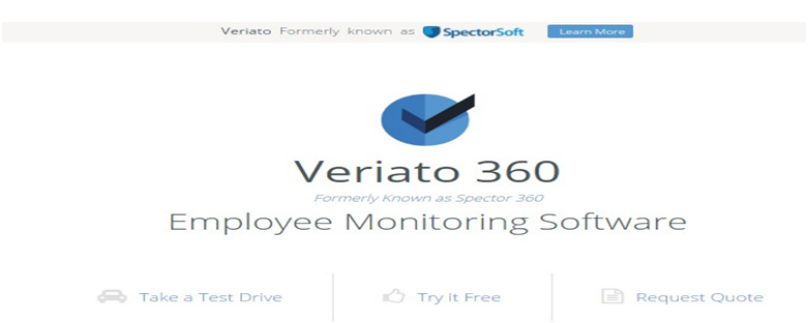

Figure 3. Veriato 360 homepage.

Veriato 360 has a different set of functionalities as compared to TSheets and ActivTrak. First, Veriato 360 has file and document tracking. This feature allows employers to view new files created and when existing files are edited, renamed or deleted. It is also useful as a supporting evidence in cases of leaks, breaches or theft. 
Second is email recording, whereby Veriato 360 records emails between clients and employees. It supports reading traditional email clients such as Outlook and also recent popular webmail such as Yahoo and Gmail. Veriato 360 allows searching across all employee emails simultaneously and sorting them according to subject, $\mathrm{CC}, \mathrm{BCC}$ and Web Mail Host columns. Finally is the keystroke logging feature. This option allows the recording of each and every thing typed on every keystroke. Applying this feature to highly positioned individuals in the company will ensure complete transparency among people with high level of access in the organization. Keystrokes can be determined on which application they are used at as well. For example, employee typing in Outlook will display in 'Outlook' and not just vaguely 'user'.

\section{Architecture and Prototype of e-Track}

The proposed project will make use of a mobile and web application architecture. Usually, a client server architecture will be applied for any form of mobile applications. However, with the development of technology and different architectures, the opportunity to take into consideration specific aspects related to mobile devices and their connectivity with servers is now available. Figure 4 shows the architecture for the proposed web-based tracking system for outfield employees, which is e-Track.

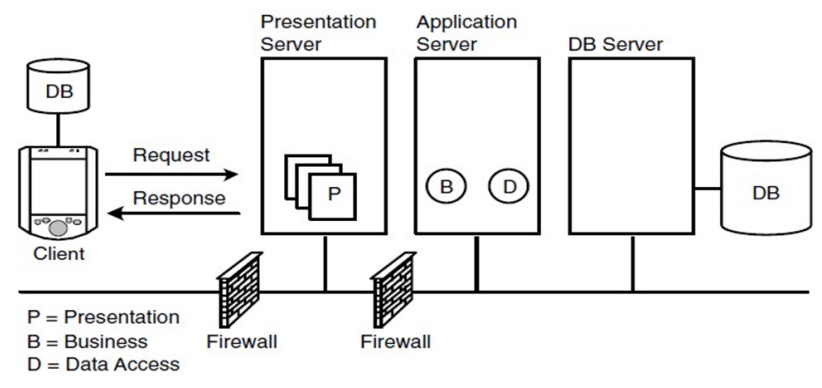

Figure 4. e-Track architecture.

Clients are divided into two categories on how they operate which are thin clients and fat clients [8]. Examples of clients are mobile device types ranging from cellular telephones, tablets and RIM devices. Thin clients do not have the ability to run custom application code therefore has to completely rely on the servers to obtain its functionality. However, the advantage is that it does not rely solely on the mobile device's operating system [9]. Thin clients uses widely available web and wireless application protocol to display html, $\mathrm{xml}$ and wml types of application content pages.

On the other hand, fat client usually has one to three layers of application code and can function without a server for a certain amount of time [8]. Practically, fat clients would be the most suitable client type to choose for businesses as even when communication between client and server is not established, the application will still be useful. For example, a fat client application will be able to accept the users input and store the date in a local database until connectivity the server is strong and the data can be transferred to the server. This feature would be essential to businesses as the user can still utilize the application even when not in contact with the server.

However, fat client does have its disadvantages. It relies heavily on the device's operating system and mobile device type. Thus, making the code tough to release and distribute. This also means the device has to support multiple code version on different devices for it to have a proper resolution (Aung, 2016). Fat client does have its variety, as it can be implemented in different ways ranging from one layer to three-layer application code. Although single layer may be useful on devices in a small scale project, it would be extremely hard to isolate individual functionalities and reuse the code in a large project. Table 1 shows the comparison between the two application code.

Nowadays, web page hosting has become a necessity in every mobile application development. This is to be able to display and service web pages using the mobile device even when the mobile client is only connected to a network and back-end system occasionally. Thus, to achieve that, the equivalent of a mini web server must be established on the mobile device.

\subsection{Prototype}

Figure 5 shows the user interface for login page. Users are required to input their own login details for their account which are email address and password. The login page has simple design and layout for users.

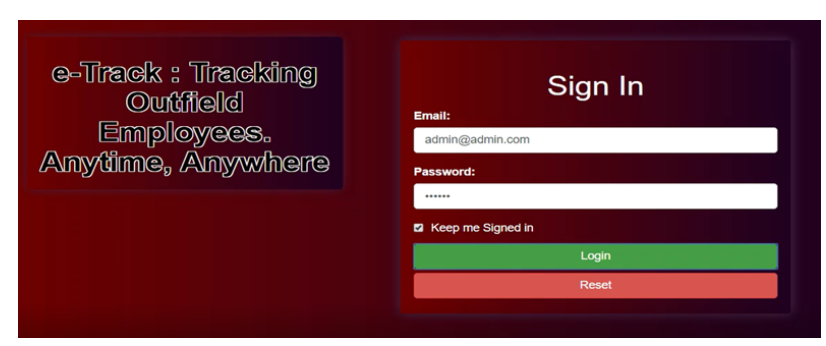

Figure 5. User interface for login.

Figure 6 shows the interface for tracking the users. Users can view the location of the employees using the google API implemented in the coding. Real-time location tracking is available when employees are logged into their accounts.

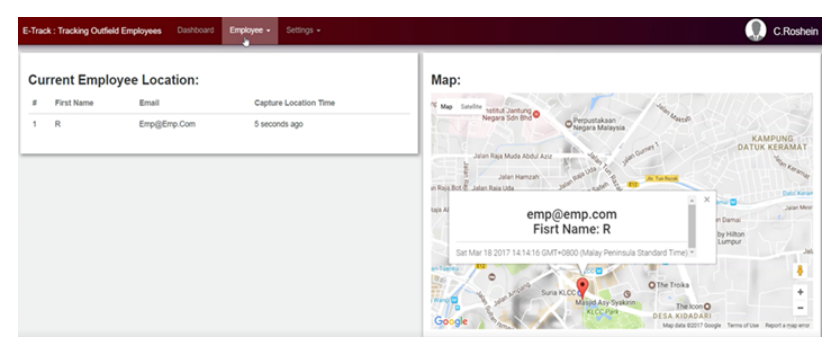

Figure 6. User interface for location. 
Table 1. Comparison between one layer to three-layer application code.

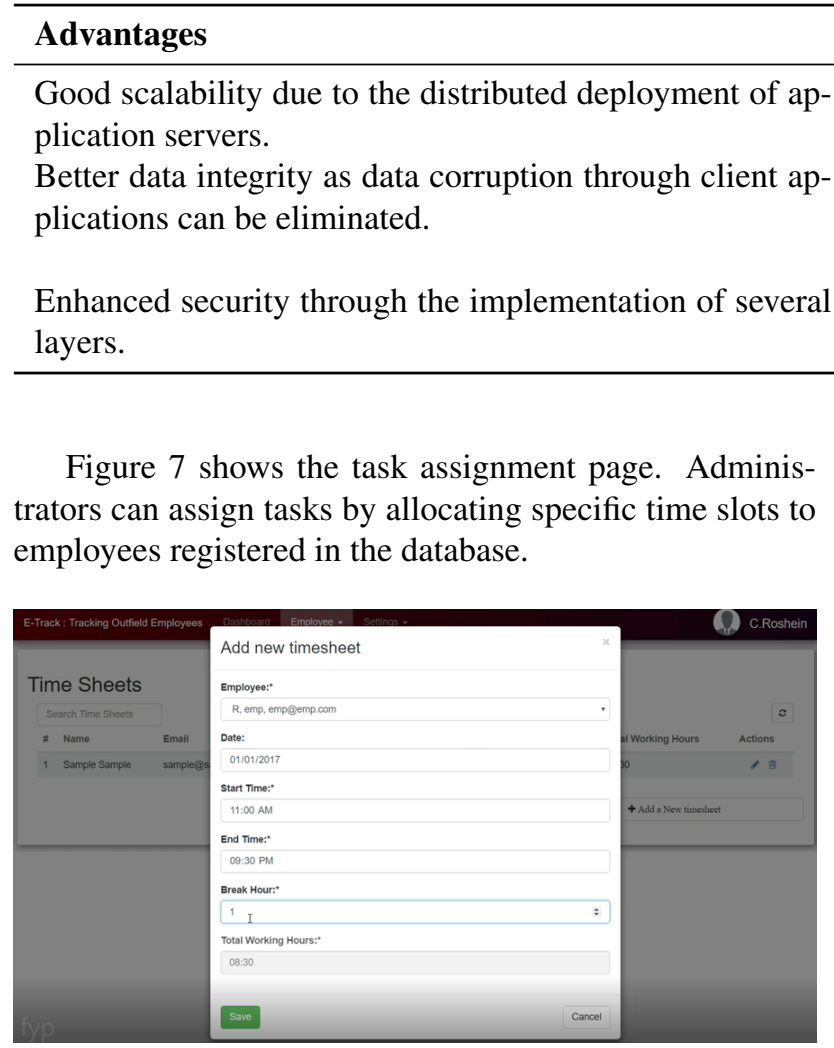

Figure 7. User interface fortask assignment.

Finally, e-Track generates list as shown in Figure 8. The users can search for specific employees and retrieve their details. The administrators will also be given power to edit information and delete employees when necessary.

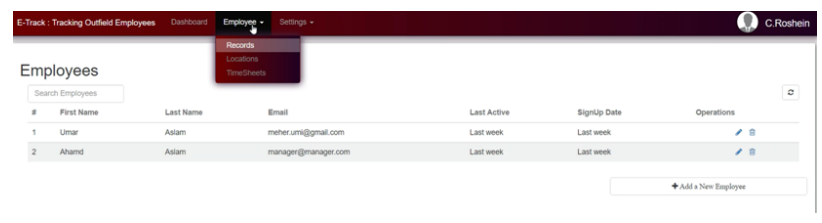

Figure 8. User interface to generate list.

\subsection{Evaluation}

e-Track was evaluated among people who work at an organization as the respondents. Questionnaires were distributed to three categories of people who are office employees, outfield employees and employees who juggle both office tasks and outfield duties. These three groups of people would be the primary users who would benefit from the usage of the system. The questionnaire was distributed through physical paper to the respondents and in order to gain a bigger pool of answers, both open-ended and close ended questions will be used. Figure 9 to Figure 13 show the evaluation findings.

Question 1: What do you think is the reason for employee productivity to decrease?
This question was done to determine the core reasons for declining employee productivity and its correlation with need of technological gadgets. The results prove that employees feel the need for the inclusion of technology to their working routing while simultaneously help address an organizations weakness.

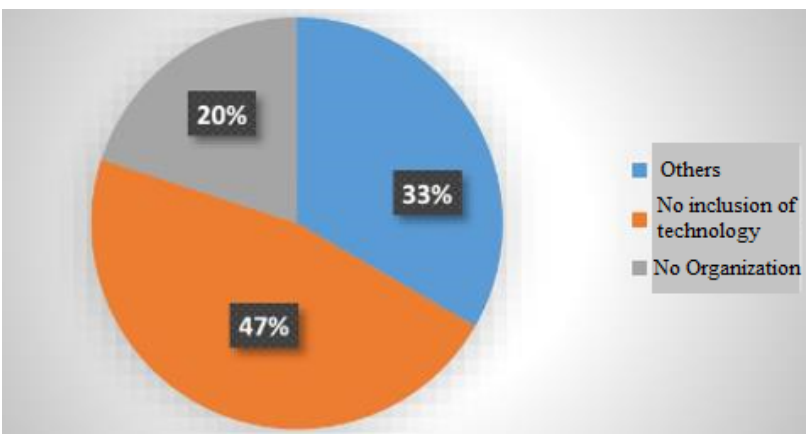

Figure 9. Findings for Question 1

Question 2: Do you do check in behalf of your colleague? If yes, why do you do it and how often?

This questions objective was to analyse if buddy punching occurs at the office place. The results show most percentage of the employees check in for their colleagues in behalf of them. This would possibly lead to a tremendous loss of revenue for any businesses.

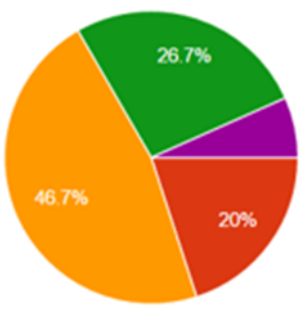

$$
\begin{aligned}
& 2 \text { to } 3 \text { times a week } \\
& \text { More than } 3 \text { time a week } \\
& \text { Once a week }
\end{aligned}
$$
- Never

Figure 10. Findings for Question 2.

Question 3: How many hours would you save in a week if you had an agile work scope?

Time equals to money. Wasted time can't be recovered especially in a business setting. This leads to the motivation behind this question. The aim was to determine how many hours can be saved or maximized by a business owner by providing their employees a flexible and agile 
work scope. The highest percentage of answers was 2 to 4 hours. These many hours per week would means 10 to 20 wasted hours in a month.

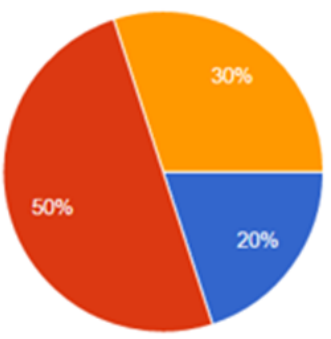

$$
\begin{aligned}
& \text { More than } 4 \text { hours } \\
& 2 \text { to } 4 \text { hours } \\
& \text { Less than an hour }
\end{aligned}
$$

Figure 11. Findings for Question 3.

Question 4: Rate the level of satisfaction of the current attendance system which is being used?

The ranges were purposefully given to over a 4 rating as the evaluation is designed so the respondents unable a neutral answer as that would not be reflective of how adaptive they are towards the current system. The results show that equal percentage of users are happy with the current manual way of handling employees. The developer believes this would be due to a phenomenon of resistance to change.

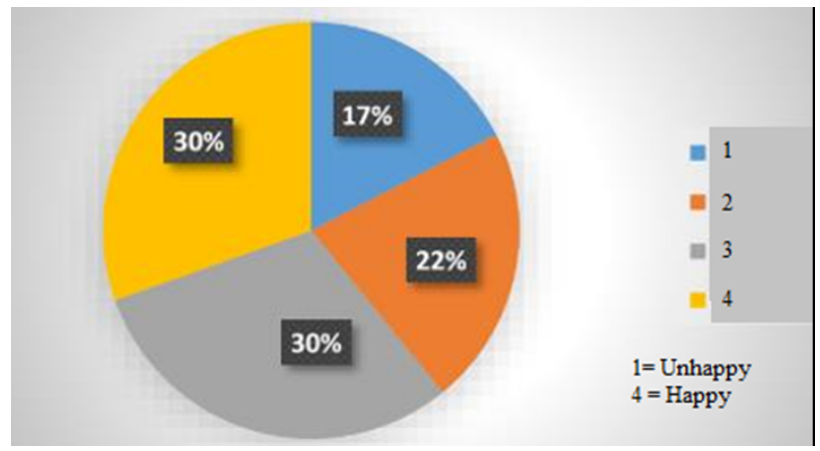

Figure 12. Findings for Question 4.

Question 5: Would you prefer if you had an agile work scope during your outfield duties where by your distances travelled will determine your payroll?

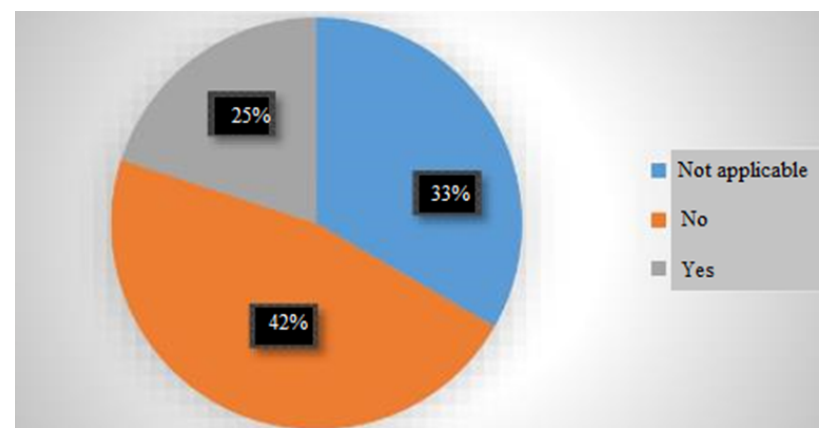

Figure 13. Findings for Question 5.
This analysis was done to decide the users requirement on if a payroll needs to be incorporated to the system for employees who travel for outfield duties. The results were mostly not receptive towards that idea. The author believes the respondents need to be further enlightened regarding the benefits of this functionality for it to be accepted wholly.

\section{Discussions and Conclusions}

The major aim of the proposed research is to design and implement a web-based tracking system for outfield employees in order to cater for various business activities as demanded by the business owners. Such demands may range from a simple task assignment, to employee location tracking and remote observation of the employees' task progress. The aim of the project is to provide both employees and business owners a convenient method to monitor business activities, employee attendance while simultaneously producing and implementing a more convenient, revolutionary method that can widen employees' work scope and increase the productivity.

The proposed e-Track System benefits both employees and business owners in a number of ways. One of the core benefit is the increased efficiency among employees. A more efficient working environment allows each employee to focus on developing their individual potential and talent in a more agile working environment. Next, the tracking system helps to reduce time wastages because employees do not have to waste unnecessary time travelling between branches during outfield duties just to sign out from their workspace. e-Track gives a time-out mechanism via a simple logout button from the mobile application.

From the perspective of business owners, the automation of the monitoring job will lessen the burden for HR department to manage their employees' whereabouts. This will enable the Human Resource (HR) department to focus on different tasks such as recruiting staffs and managing timely payrolls. A tracking mechanism based on Global Positioning System (GPS) may also reduce absenteeism cases especially when dealing with common excuses such as the traffic jam. This is because the system allow users to login from the nearest geolocation according to a specific radius required by the employer. Finally, the tracking system can also be used to locate employees during their outfield duties in case of mishappenings and emergencies.

There are also intangible benefits of the web-based outfield employee tracking system. First and foremost, employees will be able to enjoy complete freedom of workspace and improve their individual creativity in contributing to the organization, instead of being inhibited by the traditional system of having to be in the office from $9 \mathrm{am}-5 \mathrm{pm}$. Secondly, a more secure attendance check-in feature provides a more secure way to enable employees to check in to work while simultaneously reducing buddy punching. Thirdly, absenteeism can be well managed by avoiding absenteeism due to minor reasons. The system will even alert users who have not checked in to the system 5 minutes before commencement of work. The system 
also allows employees to inform beforehand failure to attend work in cases of extenuating circumstances. Finally, the application that is based on GPS location provider is more reliable and available 24/7.

Future enhancement of e-Track is to make the system more reliable in terms of calculation of employee payment based on travelled location. This will give more precise payment to employee to plan their budget before making decision. Finally, this project could be developed into smart phone application for IPhone, Android, Symbian, and Blackberry platform to use e-Track application. This application will have basic features such as trip advisor and photo gallery for front camera image capturing.

\section{Acknowledgements}

This research is sponsored by Asia Pacific University of Innovation and Technology.

\section{References}

[1] S.W. Solovic, E.R. Kadin, It's Your Biz: The Complete Guide to Becoming Your Own Boss (AMACOM Div
American Mgmt Assn, 2012)

[2] D. Preece, P. Iles, Personnel Review 38, 286 (2009)

[3] Punch card attendance system, http://www.bioenabletech.com/ punch-card-attendance-system.html

[4] T. Synerion, Retrieved May 1, 2016 (2015)

[5] Time tracking - \#1 employee time tracking software, https://www. tsheets. com/

[6] Activtrak - free employee monitoring $\mathcal{E}$ tracking software, https://activtrak.com/

[7] Veriato 360, http://www. veriato.com/

[8] W.W. Aung, Preserving green computer labs in developing countries with thin technology, in MOOCs, Innovation and Technology in Education (MITE), 2015 IEEE 3rd International Conference on (IEEE, 2015), pp. 21-25

[9] Q.F. Hassan, Innovative Research and Applications in Next-Generation High Performance Computing (IGI Global, 2016) 